\title{
Inflammatory ocular adverse events with the use of oral bisphosphonates: a retrospective cohort study
}

\author{
Mahyar Etminan PharmD MSc, Farzin Forooghian MD MSc, David Maberley MD MSc
}

\begin{abstract}
Background: There have been several published reports of inflammatory ocular adverse events, mainly uveitis and scleritis, among patients taking oral bisphosphonates. We examined the risk of these adverse events in a pharmacoepidemiologic cohort study.
\end{abstract}

Methods: We conducted a retrospective cohort study involving residents of British Columbia who had visited an ophthalmologist from 2000 to 2007. Within the cohort, we identified all people who were first-time users of oral bisphosphonates and who were followed to the first inflammatory ocular adverse event, death, termination of insurance or the end of the study period. We defined an inflammatory ocular adverse event as scleritis or uveitis. We used a Cox proportional hazard model to determine the adjusted rate ratios. As a sensitivity analysis, we performed a propensity-score-adjusted analysis.

Results: The cohort comprised 934147 people, including 10827 first-time users of bisphospho-

$\mathrm{O}$ ral bisphosphonates are the most frequently prescribed class of medications for the prevention of osteoporosis. Most literature about the safety of bisphosphonates has mainly focused on long-term adverse events, including atypical fractures, ${ }^{1}$ atrial fibrillation, ${ }^{2}$ and esophageal and colon cancer.

Uveitis and scleritis are ocular inflammatory diseases that are associated with major morbidity. Anterior uveitis is the most common type of uveitis with an estimated 11.4-100.0 cases $/ 100000$ person-years. ${ }^{4,5}$ Both diseases require immediate treatment to prevent further complications, which may include cataracts, glaucoma, macular edema and scleral perforation. Numerous case reports and case series have described an association between the use of oral bisphosphonates and anterior uveitis ${ }^{6.8}$ and scleritis. ${ }^{8.9}$ In most reported cases, severe eye pain was reported within days of taking an oral bisphosphonates, and the symptom resolved after stopping the agent. ${ }^{69}$ Only one large epidemiologic study has examined the nates and 923320 nonusers. The incidence rate among first-time users was 29/10 000 personyears for uveitis and 63/10 000 person-years for scleritis. In contrast, the incidence among people who did not use oral bisphosphonates was 20/10 000 person-years for uveitis and 36/10 000 for scleritis (number needed to harm: 1100 and 370 , respectively). First-time users had an elevated risk of uveitis (adjusted relative risk [RR] $1.45,95 \%$ confidence interval $[\mathrm{Cl}] 1.25-1.68$ ) and scleritis (adjusted RR 1.51, 95\% Cl 1.341.68). The rate ratio for the propensity-scoreadjusted analysis did not change the results (uveitis: RR 1.50, 95\% Cl 1.29-1.73; scleritis: RR $1.53,95 \%$ Cl 1.39-1.70).

Interpretation: People using oral bisphosphonates for the first time may be at a higher risk of scleritis and uveitis compared to people with no bisphosphonate use. Patients taking bisphosphonates must be familiar with the signs and symptoms of these conditions, so that they can immediately seek assessment by an ophthalmologist.
Competing interests: None declared.

This article has been peer reviewed.

Correspondence to: Mahyar Etminan, metminan@popi.ubc.ca

CMAJ 2012. DOI:10.1503 /cmaj.111752 association between the use of bisphosphonates and ocular inflammatory diseases. ${ }^{10}$ This study did not find an association, but it was limited by a small number of events and a lack of power. Thus, the association between uveitis or scleritis and the use of oral bisphosphonates is not fully known. Given that early intervention may prevent complications, we performed a pharmacoepidemiologic study to assess the true risk of these potentially serious conditions.

\section{Methods}

\section{Data sources}

We performed a retrospective cohort study using the British Columbia Linked Health Database, which is a comprehensive database with linkable data files. Physician visits are captured through the Medical Services Plan data files. The data are linkable to a comprehensive prescription drug database (PharmaNet), which captures prescription drug information, including the quantity dis- 
pensed and the number of days of medication dispensed for all residents of the province. The British Columbia Linked Health Database has been used extensively in health services and pharmacoepidemiologic research. ${ }^{11,12}$

\section{Study cohort}

The cohort included all people who visited an ophthalmologist from January 2000 to December 2007. The date of the first visit to an ophthalmologist was designated as the date of entry to the cohort. We included people who had at least one year of information about prescription drug use in the database.

We defined exposure as the first and only prescription for oral bisphosphonates after entry

Table 1: Characteristics of included patients, stratified by use of oral bisphosphonate

\begin{tabular}{|lcc|} 
& \multicolumn{2}{c|}{ No. (\%)* of patients } \\
\cline { 2 - 3 } Characteristic & $\begin{array}{c}\text { First-time } \\
\text { bisphosphonate use } \\
n=10827\end{array}$ & $\begin{array}{c}\text { No bisphosphonate } \\
\text { use } \\
n=923320\end{array}$ \\
\hline Scleritis & $392(3.6)$ & $16305(1.8)$ \\
\hline Uveitis & $185(1.7)$ & $9320(1.0)$ \\
\hline $\begin{array}{l}\text { Age cohort entry, yr, mean } \\
\text { (SD) }\end{array}$ & $68.7(12.6)$ & $51.3(22.3)$ \\
\hline Male & $2414(22.3)$ & $446889(48.4)$ \\
\hline Follow-up, yr, mean (SD) & $5.9(1.9)$ & $4.9(2.2)$ \\
\hline Sulfa-containing drugs & $822(7.6)$ & $48013(5.2)$ \\
\hline Comorbidity & $32(0.3)$ & $1847(0.2)$ \\
\hline Ankylosing spondylitis & $173(1.6)$ & $13850(1.5)$ \\
\hline Psoriasis & $108(1.0)$ & $6463(0.7)$ \\
\hline Inflammatory bowel disease & $476(4.4)$ & $18466(2.0)$ \\
\hline Rheumatoid arthritis & $1602(14.8)$ & $138498(15.1)$ \\
\hline Diabetes & $152(1.4)$ & $5539(0.6)$ \\
\hline $\begin{array}{l}\text { Systemic lupus } \\
\text { erythematosus }\end{array}$ & $22(0.2)$ & $923(0.1)$ \\
\hline Sarcoidosis & $433(0.4)$ & $3693(0.4)$ \\
\hline Multiple sclerosis & & \\
\hline $\begin{array}{l}\text { Note: SD = standard deviation. } \\
\text { *Unless otherwise stated. }\end{array}$ & & \\
\hline
\end{tabular}

Table 2: Crude, adjusted and propensity-adjusted rate ratios for the risk of uveitis and scleritis among first-time users of oral bisphosphonate

\begin{tabular}{|lccc|}
\hline Outcome & Crude RR & Adjusted* RR & $\begin{array}{c}\text { Propensity- } \\
\text { adjusted* RR }\end{array}$ \\
\hline Uveitis & $1.54(1.33-1.78)$ & $1.45(1.25-1.68)$ & $1.50(1.29-1.73)$ \\
\hline Scleritis & $1.85(1.68-2.05)$ & $1.51(1.34-1.68)$ & $1.53(1.39-1.70)$ \\
\hline
\end{tabular}

Note: $R R$ = rate ratio.

*Adjusted for age at index, sex, calendar time, use of sulfa-containing drugs, and

comorbidities (ankylosing spondylitis, psoriasis, inflammatory bowel disease, rheumatoid

arthritis, diabetes, systemic lupus erythematosus, sarcoidosis, multiple sclerosis). into the cohort. Because cases of uveitis or scleritis have been reported by first-time users of bisphosphonates, ${ }^{6,9}$ we restricted the cohort to only include first-time users, and we excluded people who had received more than one prescription. We defined nonusers as people who had not received a prescription for bisphosphonates during the follow-up period. Cohort members were followed until uveitis or scleritis developed, death, termination of health coverage, or end of the study period, whichever came first.

We obtained ethics approval from the behavioural ethics board at the University of British Columbia.

\section{Case definition}

We performed two mutually exclusive analyses with either uveitis or scleritis as the main outcome. We classified cases as people who had a first diagnosis of uveitis or scleritis. We first identified all cases using the first diagnosis of scleritis (International Classification of Diseases, ninth revision [ICD-9] code 379) or uveitis (ICD-9 code 364). We further defined cases of scleritis as those with an ICD-9 code for scleritis and a prescription for an ophthalmic or oral corticosteroid within 30 days of the diagnosis. Although patients who present with scleritis may also be prescribed nonsteroidal anti-inflammatory drugs (NSAIDs), we excluded these drugs from the main analysis because they may be prescribed for many conditions. We performed a sensitivity analysis including patients who had also received a NSAID within 30 days of the diagnosis. We defined cases of uveitis as those who had received a diagnosis of uveitis and were prescribed topical ophthalmic corticosteroids within 30 days of the diagnosis. To ensure no previous diagnoses of scleritis or uveitis had been registered in the database, we checked the period from cohort entry to the date of the outcome and looked for any previous diagnoses of either condition.

\section{Statistical analysis}

We used descriptive statistics for demographic information and the distribution of bisphosphonate users and nonusers. We built a Cox proportional hazards model to calculate hazard ratios for each study outcome. We chose a nonparsimonious approach and adjusted for the following covariates: age, sex, calendar time, history of ankylosing spondylitis, diabetes, inflammatory bowel syndrome, systemic lupus erythematosus, multiple sclerosis, psoriasis, rheumatoid arthritis and sarcoidosis. Previous studies have also adjusted for Behçet disease, Reiter syndrome, polychondritis, Wegener granulomatosis and systemic lupus erythematosus. ${ }^{10}$ However, because 
of the small number of people with these conditions in our dataset (72 in total), we excluded them from the analysis. We also adjusted for the use of sulpha-containing medications, because this is the main class of medications that has been shown to increase the risk of inflammatory eye disease. ${ }^{13}$ As a sensitivity analysis, we performed a propensity-score-adjusted analysis. We constructed a propensity-score model using the same covariables included in the proportional hazards model. We then constructed a Cox regression model, stratified on the deciles of the propensity score.

\section{Results}

From 2000 to 2007, a total of 989591 people visited an ophthalmologist. Of these, 55444 people had more than one bisphosphonate prescription; we excluded these people. Thus, we included 10827 first-time users of bisphosphonates and 923320 nonusers. Bisphosphonate users were older and more likely to be women (Table 1) compared with nonusers. The incidence rate among bisphosphonate users was 29/10 000 person-years for uveitis and 63/10 000 person-years for scleritis. Among nonusers, the incidence rate was 20/10 000 for uveitis and 36/10 000 for scleritis (number needed to harm: 1100 for uveitis, 370 for scleritis). First-time users of bisphosphonates had an elevated risk of uveitis (adjusted RR 1.45, 95\% CI 1.25-1.68) and scleritis (adjusted RR 1.51, 95\% CI 1.341.68) compared with nonusers (Table 2). The rate ratio for the propensity-score-adjusted analysis did not change the results (uveitis: RR $1.50,95 \%$ CI $1.29-1.73$; scleritis: RR $1.53,95 \%$ CI 1.39-1.70).

The results of the sensitivity analysis in which NSAID use was added to the definition for scleritis did not change the results. First-time users of bisphosphonates were still at a higher risk of scleritis compared with nonusers (adjusted RR $1.38,95 \%$ CI $1.26-1.50$ ).

\section{Interpretation}

We found that first-time users of bisphosphonates are at an increased risk of scleritis and uveitis. The sensitivity analysis did not change the results for scleritis.

In light of the reported cases of inflammatory ocular adverse events with the use of oral bisphosphonates, these conditions may be more under-reported than other adverse events that are associated with the chronic use of these drugs, mainly atypical fracture ${ }^{1}$ and cancer. ${ }^{3}$ Only one epidemiologic study has examined the risk of scleritis and uveitis with the use of oral bisphosphonates. ${ }^{10}$ French and Margo ${ }^{10}$ examined the risk of uveitis and scleritis among a cohort of US veterans with a one-year follow-up period. The relative risk of scleritis and uveitis was reported to be 1.23 among bisphosphonate users but this was not statistically significant. Despite including 35252 users of bisphosphonates, their study was limited by the small number of events, with only nine cases of uveitis and scleritis reported among first-time users. ${ }^{10}$

The reported cases of scleritis or uveitis with the use of oral bisphosphonates have mainly involved alendronate ${ }^{9}$ and risedronate. ${ }^{6}$ In most cases, symptoms occurred within days of starting bisphosphonate therapy and resolved upon stopping the drug. Reports of uveitis recurring after re-challenge with pamidronate further corroborate the causal relation, indicating that the use of bisphosphonates as a class may increase the risk of uveitis. ${ }^{14}$

The release of inflammatory mediators is believed to be the possible mechanism for bisphosphonate-induced inflammatory events. ${ }^{15}$ Alendronate and risedronate are nitrogen-based aminobisphosphonates and are considered more potent than non-aminobisphosphonates. Aminobisphosphonates have been shown to play a pivotal role in the release of tumour necrosis factor, $\alpha$-interleukin- 6 and cytokines. ${ }^{15,16}$ The surge in the concentration of inflammatory mediators, especially among new users of bisphosphonates, may put some users at a higher risk of scleritis or uveitis.

\section{Limitations}

Our study has several limitations. Because of the nature of the data, we could not verify the cases of scleritis or uveitis. Although all the cases were diagnosed by an ophthalmologist, the possibility of misclassification still exists. As with all pharmacoepidemiologic studies that use administrative data, we could only ascertain information about drug dispensing and not drug intake.

\section{Conclusion}

The results of our study are consistent with an increase in the risk of scleritis and uveitis with the use of oral bisphosphonates. Uveitis has been associated with major visual morbidity. ${ }^{17}$ The cost of vision loss in Canada was estimated to be close to $\$ 16$ billion in $2007 . .^{18}$ Bisphosphonate-induced uveitis and scleritis are potentially reversible conditions, if there is early intervention by an ophthalmologist. Although the absolute number of cases of uveitis and scleritis in this study was small, the true number of such cases among bisphosphonate users may be clinically important, given that about 5 million prescriptions for bisphosphonates are filled each year in Canada. ${ }^{19}$ 
The risk of inflammatory ocular adverse events, including scleritis and uveitis, is not highlighted in most package inserts included with oral bisphosphonates. Our study highlights the need for clinicians to inform their patients about the signs and symptoms of scleritis and uveitis, so that prompt treatment may be sought and further complications averted. Patients taking oral bisphosphonates must be familiar with the signs and symptoms of these conditions, so that they can seek immediate assessment by an ophthalmologist.

\section{References}

1. Schilcher J, Michaëlsson K, Aspenberg P. Bisphosphonate use and atypical fractures of the femoral shaft. $N$ Engl J Med 2011; 364:1728-37.

2. Heckbert SR, Li G, Cummings SR, et al. Use of alendronate and risk of incident atrial fibrillation in women. Arch Intern Med 2008; 168:826-31.

3. Green J, Czanner G, Reeves G, et al. Oral bisphosphonates and risk of cancer of oesophagus, stomach, and colorectum: case-contro analysis within a UK primary care cohort. BMJ 2010;341:c4444.

4. Mercanti A, Parolini B, Bonora A, et al. Epidemiology of endogenous uveitis in north-eastern Italy. Analysis of 655 new cases. Acta Ophthalmol Scand 2001;79:64-8.

5. Gritz DC, Wong IG. Incidence and prevalence of uveitis in Northern California; the Northern California Epidemiology of Uveitis Study. Ophthalmology 2004;111:491-500.

6. Tan YL, Sims J, Chee SP. Bilateral uveitis secondary to bisphosphonate therapy. Ophthalmologica 2009;223:215-6.

7. Malik AR, Campbell SH, Toma NM. Bilateral acute anterior uveitis after alendronate. Br J Ophthalmol 2002;86:1443.

8. Fraunfelder FW, Fraunfelder FT, Jensvold B. Scleritis and other ocular side effects associated with pamidronate disodium. Am J Ophthalmol 2003;135:219-22.

9. Mbekeani JN, Slamovits TL, Schwartz BH, et al. Ocular inflammation associated with alendronate therapy. Arch Ophthalmol 1999; 117:837-8.

10. French DD, Margo CE. Postmarketing surveillance rates of uveitis and scleritis with bisphosphonates among a national veteran cohort. Retina 2008;28:889-93.
11. Huybrechts KF, Rothman KJ, Silliman RA, et al. Risk of death and hospital admission for major medical events after initiation of psychotropic medications in older adults admitted to nursing homes. CMAJ 2011;183:E411-9.

12. Solomon DH, Massarotti E, Garg R, et al. Association between disease-modifying antirheumatic drugs and diabetes risk in patients with rheumatoid arthritis and psoriasis. JAMA 2011; 305:2525-31.

13. Fraunfelder FW. Drug-induced ocular inflammatory diseases. Drugs Today (Barc) 2007;43:117-23.

14. Ghose K, Waterworth R, Trolove P, et al. Uveitis associated with pamidronate. Aust N Z J Med 1994;24:320.

15. Hewitt RE, Lissina A, Green AE, et al. The bisphosphonate acute phase response: rapid and copious production of proinflammatory cytokines by peripheral blood gd $\mathrm{T}$ cells in response to aminobisphosphonates is inhibited by statins. Clin Exp Immunol 2005;139:101-11.

16. Thiébaud D, Sauty A, Burckhardt $\mathrm{P}$, et al. An in vitro and in vivo study of cytokines in the acute-phase response associated with bisphosphonates. Calcif Tissue Int 1997;61:386-92.

17. de Smet MD, Taylor SR, Bodaghi B, et al. Understanding uveitis: the impact of research on visual outcomes. Prog Retin Eye Res 2011;30:452-70.

18. Cruess AF, Gordon KD, Bellan L, et al. The cost of vision loss in Canada. Can J Ophthalmol 2011;46:315-8.

19. Wells GA, Cranney A, Boucher M, et al. Bisphosphonates for the primary and secondary prevention of osteoporotic fractures in postmenopausal women: a meta-analysis [Technology report no. 69]. Ottawa (ON): Canadian Agency for Drugs and Technologies in Health; 2006. Available: http://cadth.ca/media/pdf 1371_Bisphosphonates_tr_e NoAppendix.pdf (accessed 2012 Mar. 2).

Affiliations: From the Therapeutic Evaluation Unit (Etminan), Pharmaceutical Outcomes Programme, the Child and Family Research Institute, Vancouver, BC; and the Departments of Medicine (Etminan) and Ophthalmology \& Visual Sciences (Forooghian, Maberly), University of British Columbia, Vancouver, BC

Funding: This study was funded by the Canadian National Institute for the Blind.

Contributors: All authors contributed to the study conception and design, data acquisition, analysis and interpretation, and gave final approval of the version submitted for publications. 